\title{
3D Printed Machine Parts: Functionalization During Additive Fabrication
}

\author{
Daniel Gräbner ${ }^{1}$, Gerrit Dumstorff2, and Frieder Lucklum² \\ ${ }^{1}$ Friedrich-Wilhelm-Bessel-Institut Forschungsgesellschaft mbH (FWBI), D-28359 Bremen, Germany \\ ${ }^{2}$ Institute for Microsensors, -actuators and -systems (IMSAS), Microsystems Center Bremen (MCB) \\ University of Bremen, D-28359 Bremen, Germany \\ Email: graebner@fwbi-bremen.de, flucklum@imsas.uni-bremen.de
}

\begin{abstract}
:
Functionalization of machine parts by integrating sensors into the surface or bulk is necessary when we think about smart or intelligent components. So far different sensors measuring strain, temperature or e.g. corrosion have been integrated in various materials like concrete, metals or plastics. The integration is mostly done during fabrication, where the sensor is recast in the material during processing. Approaches to integrate sensors into parts fabricated by additive manufacturing are still rarely found. Especially in the case of rapid prototyping, additive techniques are going to substitute the machining of parts using classical technologies like cutting, drilling and milling. To characterize such $3 \mathrm{D}$ printed machine parts the direct integration of sensing elements is the next logical step. This can be done in multi-material printing by using insulating and conductive materials. In case of single material printing, our idea is to integrate a sensing element during the printing process itself. As proofof-concept, we present the functionalization of 3D printed screws. Strain gauges screen-printed on a $6 \mu \mathrm{m}$ thick foil are interposed into the 3D part during microstereolithography printing. We measure the torsional strain in the screw head and compare the defect effect to screws without integrated elements.
\end{abstract}

Key words: 3D printing, sensor integration, functionalized printing, smart screw, printed machine parts.

\section{Introduction}

With the recent rapid advances in additive manufacturing [1] and functional printing [2] technologies, novel types of sensor devices and parts with 'smart' components can be realized in a wide variety of materials, $[3,4,5]$. Planar microfabrication of sensor elements often results in limitations in terms of device geometry, material options, or integration challenges [6]. As alternative and addition to the use of flexible substrates [7], 3D printing of mechanical components and inkjet or screen printing of conductive and resistive layers, offers a new approach for fast, simple and inexpensive fabrication of custom designs and prototypes [8].

In previous work we have demonstrated this combination in a 3D printed pressure sensor with printed resistive read-out [9]. The vision we propose comprises sensors that can be easily integrated in any $3 \mathrm{D}$ printed component leading to smart printed systems with various integrated sensor functions. Examples are 3D printed smart devices and packaging with integrated printed sensors measuring physical parameters such as pressure, strain, temperature, or flow.

\section{Measurement Concept}

In this work, we investigate the integration of thin printed layers into 3D parts during a layer by layer printing process. For proof-of-concept, we use a screw as a standard machine part. The interesting parameter is the torsional strain between screw head and thread shaft. We embed a resistive strain gauge printed on a foil inside the screw head to correlate the torsional moment $M$ with a resistive change $\Delta R$. This allows characterization of different materials and determination of the breaking limit (Fig. 1).

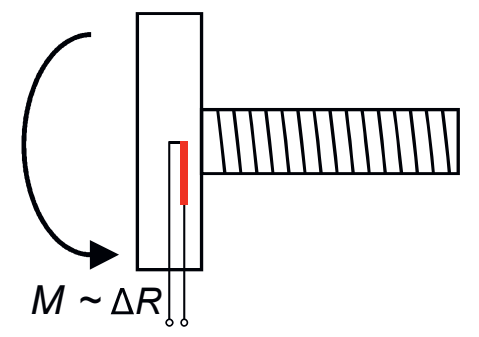

Fig. 1. Measurement concept of 'smart' screw. 


\section{Screen-Printed Strain Gauge Foil Sensor}

The design of the strain gauge is shown in Fig. 2(a). Two strain gauges of a carbon based ink are printed on a foil and connected by silver printed tracks. Because silver inks have a much lower resistance than carbon based inks, the lead resistance of the strain gauges can be neglected. The width of the strain gauges and the electrical path is $400 \mu \mathrm{m}$. The strain gauges can be connected in a Wheatstone bridge circuit as a half-bridge. When the screw is set under a torsional moment, the inner strain gauge is for temperature compensation and it underlies almost zero strain because it is close to the neutral fiber of the screw. The outer strain gauge is set to tensile strain due to the torsional moment. Electrical tracks lead from the strain gauges to the contact pad area (see also Fig. 1), where they are connected with wires.

Functional printing is processed on a manual screen printing machine with a low cost plastic screen from SEFAR (Type PCF FC 180-27-Y$P W$ ). The substrate is a $6 \mu \mathrm{m}$ thick polyester foil (Mylar), which is stretched on a ceramic carrier. A relatively thin foil is necessary because the slice height of the 3D printer is $25 \mu \mathrm{m}$ (see next section). In the first step, the electrical tracks are printed with the silver filled, stretchable conductor paste PE873 from DuPont. The structure is cured for $20 \mathrm{~min}$ at $120^{\circ} \mathrm{C}$ in an oven. The film thickness after drying is around 5 $\mu \mathrm{m}$. In the second step the carbon based ink ECI 7004LR E\&C from Henkel is applied. Afterwards the strain gauges are electrically connected at the contact pad area by a copper wire and electrically conductive adhesive (Panancol Elecolit 3653 , curing at $80^{\circ} \mathrm{C}$ in an oven). The final foil sensor is shown in fig. 2(b).

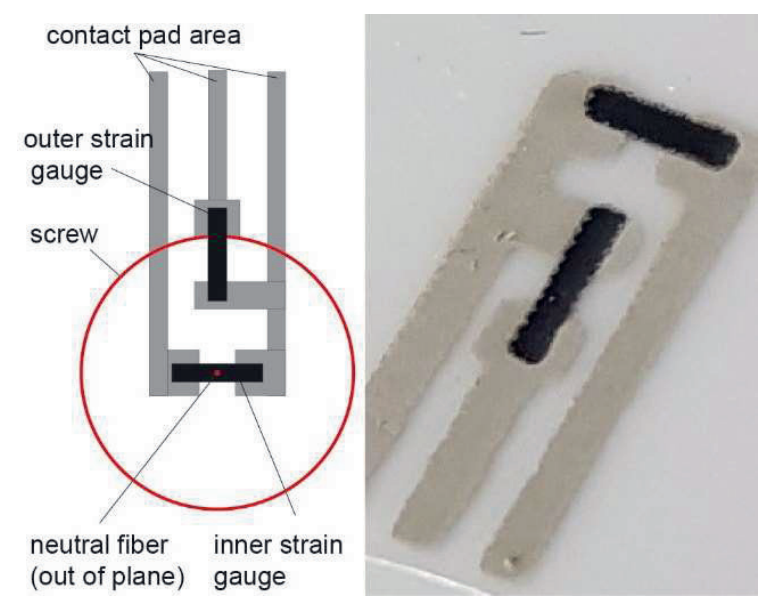

Fig. 2. a) Strain gauge layout relative to screw shaft, (b) screen-printed strain gauges on $6 \mu \mathrm{m}$ thick polyester foil.

\section{D Printed Screw}

As proof-of-concept machine part, we have designed an ISO M6 screw with hex-head and washer disk. The CAD model has been created in Autodesk Inventor. It consists of an M6 thread created by cutting a thread profile $\left(60^{\circ}\right.$ flank angle, slightly enlarged from ISO norm) into a $15 \mathrm{~mm}$ high screw shaft. A $1 \mathrm{~mm}$ thick, $15 \mathrm{~mm}$ wide spacer disk, and a $5 \mathrm{~mm}$ thick hexagon block for a $10 \mathrm{~mm}$ screw wrench form the screw head. For sensor foil integration we remove two $4 \mathrm{~mm}$ wide, $0.5 \mathrm{~mm}$ deep, opposing contact pad regions, either on top or bottom face of the spacer. We also add three holes each side for easier handling of wire contacts. Finally, some labels are engraved on top and bottom face. The final model is shown in Fig. 3.

As alternative, we have also designed and fabricated M8 screws, as well as working with various other sizes and norms as small as ISO M3 or UNC \#2-56. To account for fabrication tolerances, as well as shrinkage after printing, each thread profile, including minor and major diameter have to be slightly adjusted. This adjustment is highly dependent on the type of printing material, but also on processing and post-processing parameters such as exposure time and UV curing.

All parts are fabricated in an upright orientation using a microstereolithography printer (Perfactory Micro HiRes, EnvisionTec Inc., USA) at a slicing height of $25 \mu \mathrm{m}$. Three identical screws are printed in one run, two for foil integration and one as reference without foil. We utilize two different acrylic resin materials. The first is a high resolution, high temperature, low viscosity resin (HTM140 M, EnvisionTec Inc.) exposed for $3000 \mathrm{~ms}$ per slice, which

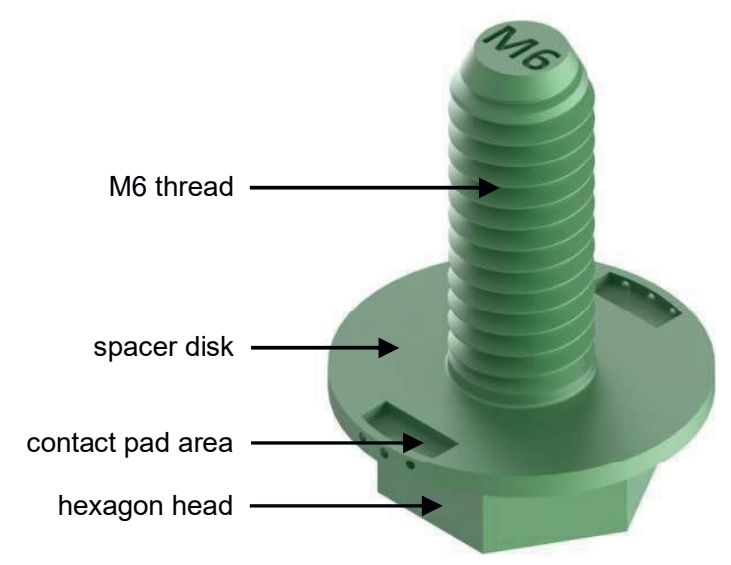

Fig. 3. 3D CAD model of M6 screw with hexagon head, spacer disk, and contact pad opening for sensor foil integration. 
results in a hard and tough, but brittle plastic with superior details and surface finish. The other is considerably more viscous, sticky resin (ABS Flex, EnvisionTec Inc.), which results in a flexible, elastic plastic with softer surface finish but less fine details. Here, we choose an exposure time of $800 \mathrm{~ms}$ per slice. The printing direction starts with the hex-head and washer disk based on automatically generated supports.

\section{Sensor Foil Integration}

For integration of the sensor foil element, we pause the print job after reaching a height of half the spacer disk (after $0.5 \mathrm{~mm}$ thickness). The printing platform is removed from the printer and carefully cleaned of excess resin. We have looked at two different integration states, leaving the resin film that covers all surfaces or rinsing all parts with isopropanol and drying the surfaces. In both cases, we place the sensor foil manually onto the washer disk, with the contact pads oriented upwards or downwards depending on the orientation of the contact cavity. The foil is centered and aligned according to the desired position of the resistors. Due to the strong adhesion of the thin foil to any surface, no further securing steps are necessary. The printing platform is then carefully realigned into its initial position and the printing process is resumed.

After printing, all parts are post-processed in a standard fashion, rinsing and cleaning with isopropanol, drying with compressed air, and post-curing in a flood UV bath for 2-5 minutes. Depending on the type of screen-printed ink that is exposed at the contact pads, compatibility with this process, such as chemical resistance to the liquid resins and isopropanol, stability during ultrasonic cleaning has to evaluated. Some post-processing steps such as ultrasonic cleaning can be adjusted or skipped.

Finally, each 'smart screw' requires electric contacts to read out the strain gauge resistors. We connect thin wires through the printed alignment holes to the contact pads using electrically conductive glue or low temperature solder. It is also advantageous to encapsulate the contact region with non-conductive glue or resin. If this region is opened towards the thread, the encapsulation should not extend beyond the washer thickness.

A photograph of an early prototype sample is given in Fig. 4. The integrated foil sensor is visible through the half thickness of the spacer disk and challenges of alignment and electrical contact are still evident. Printed samples of both materials work well with metal and plastic nuts.

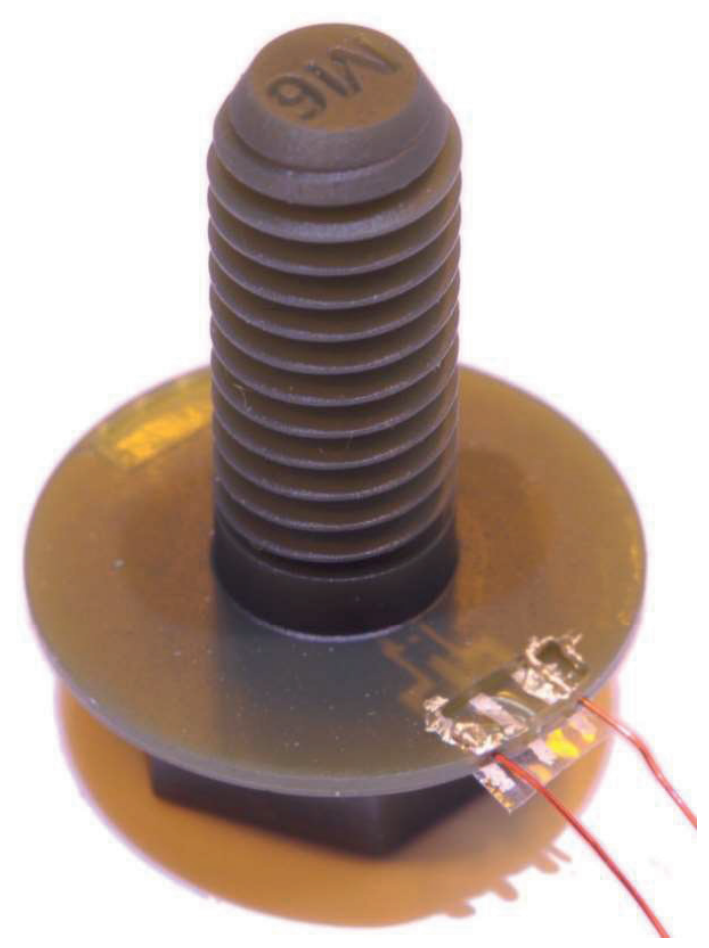

Fig. 4. Photograph of early printed screw prototype with integrated sensor foil element; some misalignment and challenges of electrical contacts are still evident.

\section{Mechanical Characterization}

For compatible combinations of inks, resin, and conductive glue, we have observed no or only negligible strain due to the integration process. While we expect a compressive load on the resistors, the low thickness of foil and film, as well as the layer by layer printing does not lead to a noticeable change of the resistances. The foil is firmly embedded between the printed layers.

We performed die shear testing of the screws with and without integrated sensors to determine the influence of the foil on the mechanical properties of the screw. Fig. 5 shows typical die shear test results for the two types of materials. The hard and brittle HTM140 M screws with as well as without integrated foil typically shear off at the shaft, indicating no negative influence of the integrated sensor on the mechanical reliability. The more elastic ABS Flex screws shear off at the shaft as well, if there is no sensor integrated. The ABS Flex screws with integrated sensor always shear off right above the foil, meaning that the mechanical durability of these screws is strongly deteriorated by an integrated sensor. The most likely reason for this influence is a poor adhesion between the ABS Flex material and the foil. Pausing, cleaning and resuming the printing process can also be another influencing factor. 


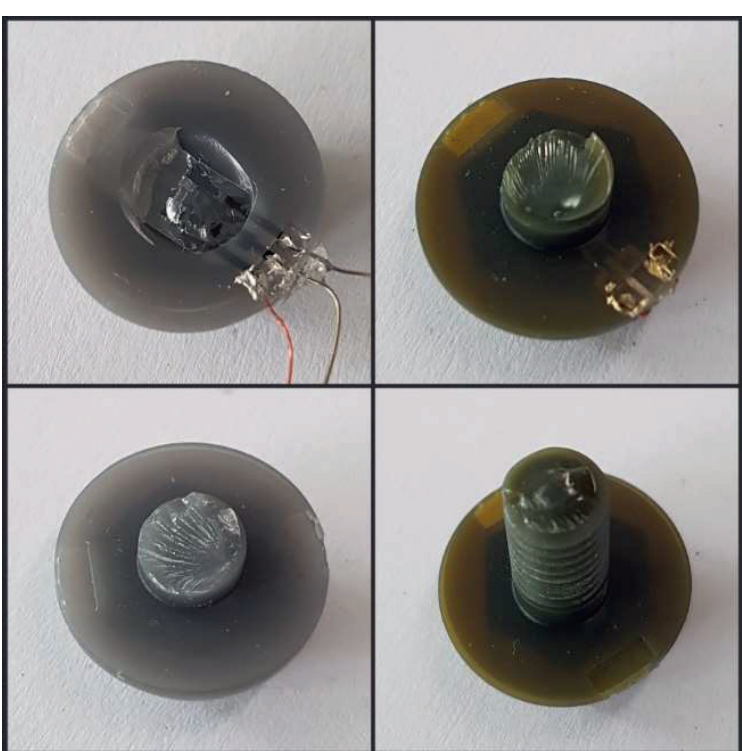

Fig. 5. Photographs of sheared off screws: ABS Flex Screw with integrated sensor (top left), $A B S$ Flex Screw without integrated sensor (bottom left), HTM140 M screw with integrated sensor (top right, HTM140 M screw without integrated sensor (bottom right).

\section{Measurement Results}

The measurement setup is shown in Fig. 6. An M6 nut is fixed on the edge of the work piece holder. A teflon plate is used to create a fixed distance between the screw head and the nut. A displacement at a specified push speed are applied to the wrench with the multitesting tool XYZTEC Condor 100. Applied force over

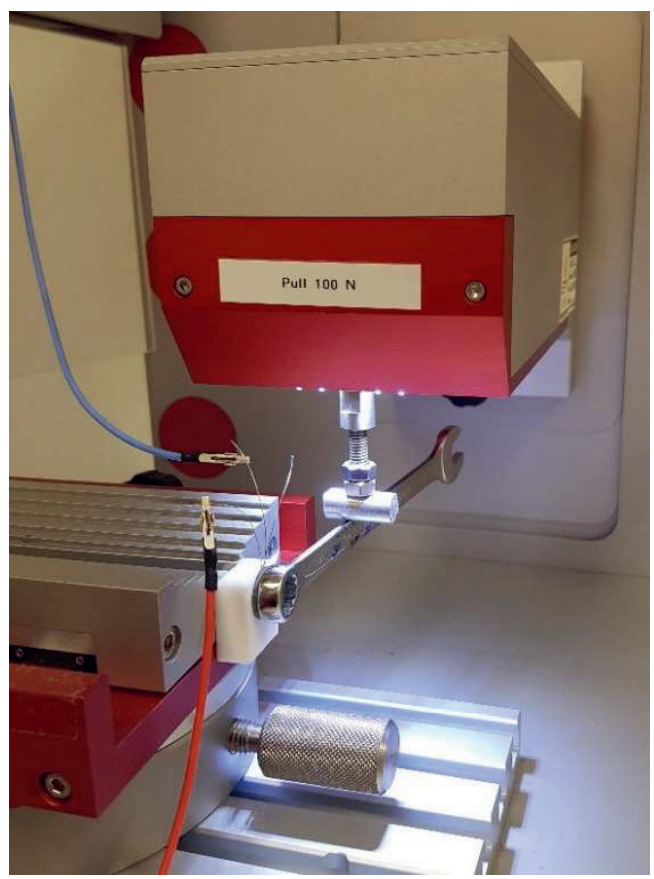

Fig. 6. Measurement setup consisting of push/pull tester recording applied force of displacement, a screw wrench fastened to the screw head, and a nut fastened to screw thread and clamped into a holder.

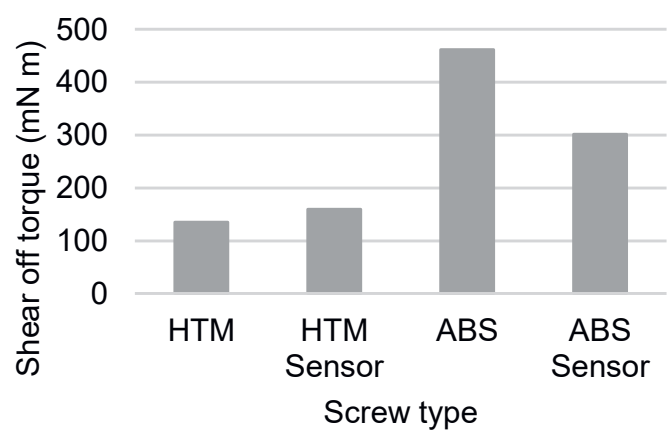

Fig. 7. Die shear test results for the different plastic materials with and without embedded sensor element.

distance is recorded. The screw wrench has distance marks on it, so the lever arm length can easily be determined in order to calculate the resulting torque on the screw. The integrated strain gauges change in resistance is monitored by a Keithley 2000 multimeter while fastening the screw.

During shear testing, the torque necessary to shear off the screws was recorded. The results are shown in Fig. 7. The HTM140 M screws with and without sensor only show a negligible difference in shear off torque around $140 \mathrm{mNm}$. the shear off torque for ABS Flex screws without sensor is considerably higher at $450 \mathrm{mNm}$ than for those screws with embedded sensor at $300 \mathrm{mNm}$. The results correspond with the previously shown shear off behavior.

As an example of the sensor functionality, the electrical response of the strain gauge in a screw made from HTM140 M is ilustrated here. Fig. 8 shows the relative change in resistance versus the torque applied to the screw head. The resistance increases linearly with the torque after the screw is fastened. We calculate a sensitivity of $32 \frac{\%}{\mathrm{Nm}}$ from the results.

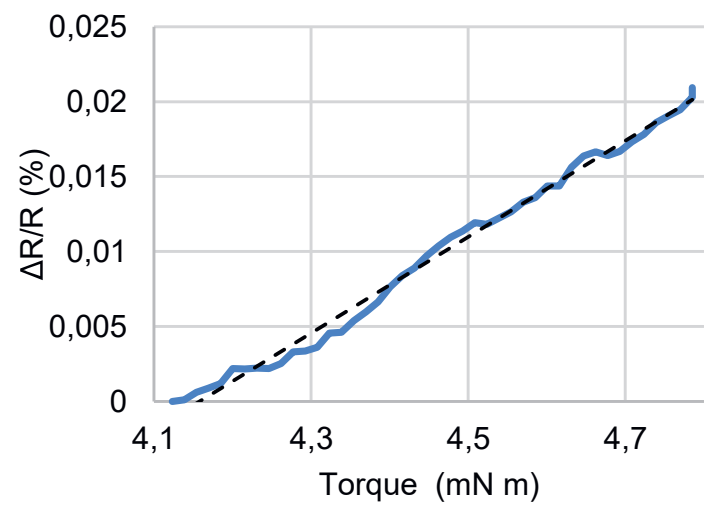

Fig. 8. Relative change of resistance versus torque applied on an HTM140 M screw head after the fastening phase. 


\section{Conclusions}

We have presented the fabrication of a 3Dprinted screw with an integrated resistive strain gauge made by functional screen printing. For integration, the layer by layer 3D printing process was paused, a foil sensor embedded onto the surface, and printing resumed. The screws were manufactured from two different materials: HTM140 M, which is hard and brittle, and ABS Flex, which is more flexible. The influence of the integrated sensor on the mechanical behavior has been evaluated by die shear testing. The HTM140 M screws are not negatively influenced by the integration, whereas the mechanical durability of the ABS Flex screws is strongly deteriorated by the integrated sensor. The reason for the strong deterioration is most likely the poor adhesion between ABS Flex material and the sensor foil.

The relative change in resistance of the integrated strain gauges while applying torque on a HTM140 M screws head has been recorded. The strain gauge is able to measure the torsional strain in the screws head with a sufficient sensitivity of $32 \frac{\%}{\mathrm{Nm}}$.

\section{Acknowledgment}

The authors thank Ingrid Michels for assistance with electrical contacting of printed sensor foils. The authors also acknowledge the University of Bremen for funding the postdoctoral research project "AddSense: Additive manufacturing for local fabrication of sensor structures for material integrated sensing".

\section{References}

[1] M. Vaezi, H. Seitz, and S. Yang, "A review on 3D micro-additive manufacturing technologies", Int. J. Adv. Manuf. Technol. 67, 1721-1754, 2013.

[2] S.-J. Woo et al., "A thin all-elastomeric capacitive pressure sensor array based on micro-contact printed elastic conductors," J. Mater. Chem. C, vol. 2, pp. 4415-4422, 2014.

[3] Lehmhus, D., Aumund-Kopp, C., Petzoldt, F., Godlinski, D., Haberkorn, A., Zöllmer, V., \& Busse, M. (2016). Customized Smartness: A Survey on Links between Additive Manufacturing and Sensor Integration. Procedia Technology, 26, 284-301.

[4] Delamare, John, Remco Sanders, and Gijs Krijnen. "3D printed biomimetic whisker-based sensor with co-planar capacitive sensing." SENSORS, 2016 IEEE. IEEE, 2016.

[5] Leigh, S. J. "Polymer composites for 3D printing of functional sensors and transducers." SENSORS, 2016 IEEE. IEEE, 2016.

[6] G. Dumstorff, S. Paul, and W. Lang., "Integration Without Disruption: The Basic Challenge of Sensor Integration," IEEE Sensors J., vol. 14, no. 7, pp. 2102-2011, 2014.

[7] K. Lee et al., "Carbon nanotube film piezoresistors embedded in polymer membranes," Appl. Phys. Lett., vol. 96, no. 19, p. 013511, 2010.

[8] D. Espalin, D. W. Muse, E. MacDonald, R. B. Wicker, "3D Printing multifunctionality: structures with electronics," Int. J. Adv. Manuf. Technol., vol. 72, no. 5, pp. 963-978, 2014.

[9] F. Lucklum, G. Dumstorff, "3D Printed Pressure Sensor with Screen-Printed Resistive Read-Out", Proc. IEEE Sensors 2016, Orland, FL, Oct. 2016. 